\title{
Tra intimità e ricerca di sé: Pia Epremian e il cinema sperimentale in Italia tra gli anni Sessanta e Settanta
}

\author{
Maria Alicata \\ Sapienza Università di Roma \\ maria.alicata@gmail.com
}

RIASSUNTO: II presente saggio intende approfondire alcuni aspetti specifici della filmografia di Pia Epremian De Silvestri film-makernata a Chivasso in provincia di Torino nel 1942. Attraverso l'analisi dei suoi film è possibile ripercorrere la vicenda del cinema sperimentale in Italia che fiorisce nella seconda metà degli anni Sessanta epoca che vede nascere spazi di condivisione e collaborazione: collettivi di artisti, di cineasti come la Cooperativa Cinema Indipendente (1967-1971), di studenti, di femministe. Tra le poche donne film-makers attive in quel periodo, i suoi lavori rispecchiano la ricchezza di questo momento storico tra produzione e sperimentazione artistica, dove la dimensione soggettiva e personale è predominante. Si tratta di un cinema girato in prima persona e che ha contribuito alla creazione di una nuova identità femminile.

PAROLE CHIAVE: Epremian; Cinema d'artista; Cinema Underground; Corpo; Performatività; Cooperativa Cinema Indipendente; Donne film-maker.

\section{Between Intimacy and Search of the Self: Pia Epremian and Experimental Cinema in Italy between the Sixties and Seventies}

ABSTRACT: The essay intends to analyze some specific aspects of the filmography of Pia Epremian De Silvestri, a film-maker born in Chivasso in the province of Turin in 1942. Through the analysis of her films it is possible to retrace the story of experimental cinema in Italy that flourished in the second half of the Sixties. At this time new spaces of sharing and collaboration were born including collectives of artists, students, feminists and filmmakers such as the Independent Cinema Cooperative (1967-1971). Among the few women filmmakers active at that time, Empremian's works reflect the richness of this historical moment between production and artistic experimentation, where the subjective and personal dimension is predominant. This is a cinema shot in first person that characterizes its production and that has contributed to the creation of a new female identity.

KEYWORDS: Epremian; Art films; Underground cinema; Body; Peformativity; Independent Film Cooperative; Women filmakers.

Recibido: 28 de febrero de 2018 / Aceptado: 8 de enero de 2019.

Questo saggio vuole approfondire alcuni aspetti specifici della filmografia di Pia Epremian De Silvestri, film-maker ${ }^{1}$ nata a Chivasso in provincia di Torino nel 1942.

Attraverso l'analisi dei suoi film è possibile ripercorrere la vicenda del cinema sperimentale che fiorisce in Italia nella seconda metà degli anni Sessanta, epoca che vede nascere spazi di condivisione e collaborazione: collettivi di artisti e cineasti come la Cooperativa Cinema Indipendente (1967-1971), di studenti, di femministe. Tra le poche donne film-maker attive in quel periodo, i suoi lavori rispecchiano la ricchezza di questo momento storico tra produzione e sperimentazione artistica, dove la dimensione soggettiva e personale è predominante. Questa produzione, caratterizzata da un cinema girato in prima persona, ha saputo generare nuove forme di rappresentazione che hanno contribuito alla creazione di una nuova identità femminile (Mulvey, 1989).

Dopo la laurea in lettere e filosofia nel 1967, Pia Epremian frequenta da subito il contesto della scena artistica di Torino insieme all'amico di infanzia e collega il regista Tonino De Bernardi che prenderà spesso parte ai suoi film. Per meglio com-

Cómo citar este artículo: ALICATA, Maria, "Tra intimità e ricerca di sé: Pia Epremian e il cinema sperimentale in Italia tra gli anni Sessanta e Settanta», Boletín de Arte-UMA, n. ${ }^{\circ}$ 40, Departamento de Historia del Arte, Universidad de Málaga, 2019, pp. 245-253, ISSN: 0211-8483, e-ISSN: 2695-415X, DOI: http://dx.doi.org/10.24310/BoLArte.2019. v0i40.5703 
prendere la sua formazione, le basi critiche e teoriche della sua produzione, è importante fornire un quadro del contesto artistico e culturale in cui si inserisce tale esperienza.

La vicenda del cinema sperimentale italiano è doppiamente legata al movimento delle neoavanguardie artistiche e al periodo della grande fertilità culturale e politica negli anni Sessanta in Italia. Tutta la scena italiana è vivace, ma è soprattutto a Roma e Torino che si cristallizza una comunità di artisti che esprimono nuovi percorsi nei linguaggi, rompono con la tradizione del dopoguerra, guardano alle avanguardie storiche (dada e futurismo) e alle trasformazioni di una società nel pieno del boom economico ${ }^{2}$. La macchina da presa, allo stesso tempo strumento e dimensione dell'immaginario, diviene il mezzo per agire in una prospettiva intermedia, di passaggio liminale tra le arti, tra l'arte e la vita, e per la ricerca di una nuova posizione dell'artista rispetto alla realtà sociale. Pittori, poeti, registi e autori di teatro, cineasti, compositori, realizzano in questo periodo delle azioni unite da un nuovo intreccio di linguaggi e di mezzi tecnologici.

Gli artisti sentono il bisogno di avvicinarsi alla cultura dei massmedia sconfinando tra arte e cinema. Nasce così il cinema d'artista ${ }^{3}$ diverso nella forma e nei contenuti da quello commerciale, pronto a trasgredire ogni regola della mercificata società di massa, poiché non cerca ad ogni costo il consenso del grande pubblico.

Nel maggio del 1967 la Galleria d'Arte Moderna di Torino organizza una presentazione del New American Cinema che dava risalto, come indiscutibile protagonista del nuovo fronte sperimentale di ricerca, al ricco repertorio di film realizzati dal regista Jonas Mekas (Fagone, 2008). La manifestazione, promossa dalla Unione culturale di Torino e curata da Aldo Passoni, aveva coinvolto e entusiasmato amatori del cinema e delle arti visuali, colpiti dalla lucida consapevolezza e capacità comunicativa di Jonas Mekas e dai suoi film profondamente innovativi realizzati a partire dalla seconda metà degli anni Cinquanta. Nel cinema sperimentale di Jonas Mekas si realizza l'inedita prospettiva di un «cinema differente» (Fagone, 2008: 13) che non si cura delle regole e delle funzioni dominanti della cinematografia, meno preoccupato del destinatario che dell'autore, del referente che del messaggio stesso, del principio di realtà, del simbolico che dell'iconico, in breve, meno preoccupato del senso che della forma (Fagone, 2008: 13). Si tratta di film radicalmente personali, che si rapportano prevalentemente, nella concezione, nella rea- lizzazione e nella diffusione, all'arte e all'artigianato (non al commercio e all'industria) ${ }^{4}$.

Per i cineasti italiani, già in contatto tra loro, le condizioni sono mature per lo sviluppo della ricerca e della produzione di un linguaggio autonomo che si distingue per un maggiore realismo. Registi come Piero Bargellini, Adamo Vergine, Tonino De Bernardi, la stessa Pia Epremian, assimilata la «non-lezione» di Mekas, realizzano delle opere di una innovazione e di una forza espressiva fino a quel momento sconosciute. La loro totale refrattarietà al mercato e alle sue dinamiche, ad una professionalizzazione sia dell'arte che del cinema, così come la dimensione privata ed esistenziale del loro approccio cinematografico, rappresentano le caratteristiche più originali di questa stagione creativa. E' importante inoltre ricordare che, accanto ad essi, si stava sviluppando in Italia una sperimentazione artistica che poneva su un piano linguistico innovativo l'intreccio di arte e immagine in movimento, film di artisti (non di cineasti sperimentali) che operano con la cinepresa si datano già al $1960^{5}$.

Le produzioni di questi anni si caratterizzano soprattutto per due aspetti: la determinazione linguistica molto libera, diretta ed efficace; l'autonomia e l'indipendenza dalle strutture condizionanti della produzione cinematografica commerciale. Quanto scaturisce da questa sperimentazione è un'inedita e produttiva possibilità di utilizzare in senso creativo le risorse comunicative ed espressive sulle quali la macchina da presa opera principalmente: la nozione del tempo, la memoria e la simultaneità. Ne conseguono un'immediatezza che consente di presentare un'immagine ripresa non convenzionalmente senza sottoporla a nessun tipo di trattamento, oltre a diverse modalità di scomposizione o ricomposizione della visione: tutti elementi che contribuiscono alla creazione di una dimensione temporale e narrativa del tutto nuova.

Al di là di questo importante incontro con l'esperienza della avanguardia americana, più volte citato dalla stessa Epremian ${ }^{6}$ e innestato su una pratica sperimentale che in Italia aveva già avuto importanti esiti, occorre ribadire come la città di Torino sia il "campo sensibile» (Celant, 1976: 10) in cui si sviluppa il contesto favorevole alla nascita dell'Arte Povera, il movimento artistico profondamente interdisciplinare che si è aperto al teatro, alla performance e al cinema.

Roma e Torino in quegli anni sono gli assi geografici in cui si sviluppano le più interessanti ricerche artistiche del periodo, dove, in un contesto favorevole alla sperimentazione, 
il mezzo cinematografico viene utilizzato dagli artisti in uno stretto dialogo tra le cosiddette time-based media e le arti visive. II cinema, strumento prezioso, specialmente nella forma leggera, duttile, economica del piccolo formato, utilizzato anche come dispositivo di documentazione e di denuncia, è scelto come medium per dare corpo a quel "partire da sé» che traduceva, nel linguaggio di allora, il passaggio dall'individuo alla collettività. In una tale prospettiva dove il confine tra arte e vita diviene estremamente sottile, quello di opera d'arte totale è un concetto utile per interpretare la situazione sociale del momento (Willener, 1970). Tale nozione si rivela altrettanto significativa nell'analisi della coeva dimensione estetica: «totalità» dunque, come tensione a superare le separazioni «tra fine e mezzo ([...]) tra immagine e azione, tra conoscenza e vita» (Perniola, 2009: 42-43).

A Napoli, nel maggio del 1967, sul modello della Film-Maker's Cooperative di New York, sorta intorno alla figura carismatica di Jonas Mekas, viene fondata la Cooperativa Cinema Indipendente con il fine di aggregare gli autori del cinema sperimentale e distribuirne i film, dapprima a Napoli e poi anche a Roma e Torino. Per la maggior parte si tratta di autori romani o di Torino; Pia Epremian è l'unica donna a far parte del primo catalogo (ad esclusione di Anna Lajolo, che tuttavia firma tutte le sue opere assieme al marito Guido

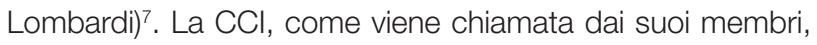
ha da subito una connotazione non solo culturale ma anche politica nelle modalità di sperimentare, produrre e distribuire. Nel programma ciclostilato di una rassegna tenutasi presso il Filmstudio di Roma dal 12 al 21 marzo 1969 intitolata «Cinema underground italiano", si può leggere:

La Cooperativa Cinema Indipendente è un'ipotesi di lavoro. Un gruppo di amici, perché no? Chiunque abbia un pizza sottobraccio può aderire. [...] Questi film sono fatti soprattutto per essere visti. non vi è ragione perché qualche spettatore particolarmente entusiasta o volenteroso non contribuisca in un modo qualsiasi alla diffusione di tutto questo amore ( $\mathrm{Di}$ Marino, 1999).

Dopo questa premessa storica, è importante introdurre un altro elemento chiave della creazione artistica di questi anni: il corpo.

Sono proprio le pratiche incentrate sul corpo che contribuiscono a mettere in atto il graduale ma fondamentale cambiamento di paradigma che si è verificato nella seconda metà del secolo scorso nella articolazione del soggetto nella società (Jones, 1998: 19). Parlare di corpo come comportamento nell'arte a partire dagli anni Sessanta del Novecento, implica considerare come questo sia stato simultaneamente al centro di pratiche artistiche, di rivolte interiori e provocazioni politiche: il corpo agisce, è artefice di azioni. L'azione diviene un modo per riflettere sul corpo e i comportamenti, sui codici e le norme alla base di concezioni e definizioni culturali e sociali.

Al «corpo come linguaggio» (Vergine, 2000), citando il fondamentale testo del 1974 di Lea Vergine, ricorrono infatti in quegli anni molti artisti delle più disparate tendenze e differenti tecniche: «Registratori, cineprese, macchine fotografiche sono i mezzi impiegati per fermare una quantità di piccoli episodi privati. L'artista diventa il suo oggetto, pone sé stesso come oggetto, essendo cosciente di tale processo» (2000: 15). E ancora vi sono «riscontrabili dei caratteri che fanno da comune denominatore a questa maniera di fare arte, come: la perdita di identità, il rifiuto del prevalere del senso della realtà sulla sfera emozionale, la romantica ribellione alla dipendenza da qualcosa e da qualcuno, la tenerezza mancata e quindi frustrante; l'assenza (e l'angoscia che ne deriva) di una forma «adulta» altruistica d'amore» (2000: 7).

Proprio, dunque, nel punto di incontro tra sperimentazione cinematografica e una specificità legata al «corpo come linguaggio» si colloca il cinema di Pia Epremian: sono queste le tematiche che possono essere identificate attraverso l'analisi dei suoi film.

La produzione della film-maker si concentra in pochi anni, dal 1967 al 1970, periodo in cui realizza nove film di breve e media durata, tutti in $8 \mathrm{~mm}$. È da notare come la pellicola in $8 \mathrm{~mm}$ sia la più utilizzata in quel periodo proprio perché era il formato più economico; il 16 millimetri era già troppo costoso, come narrato anche da Tonino De Bernardi (De Bernardi, 2017). Oltre ad essere il mezzo più accessibile, il piccolo formato veniva impiegato per la ricerca e la sperimentazione, campi in cui offrì i migliori risultati per rappresentare le fantasie ed esprimere la creatività degli autori.

Pia Epremian esplora, in un'originale miscela di performance e home movie, dei possibili spazi di libertà personale. Nei suoi film la regista restituisce fisicità al corpo femminile facendolo uscire dalle cornici culturali in cui era stato confinato, inserendosi in quel percorso intrapreso dagli artisti a 


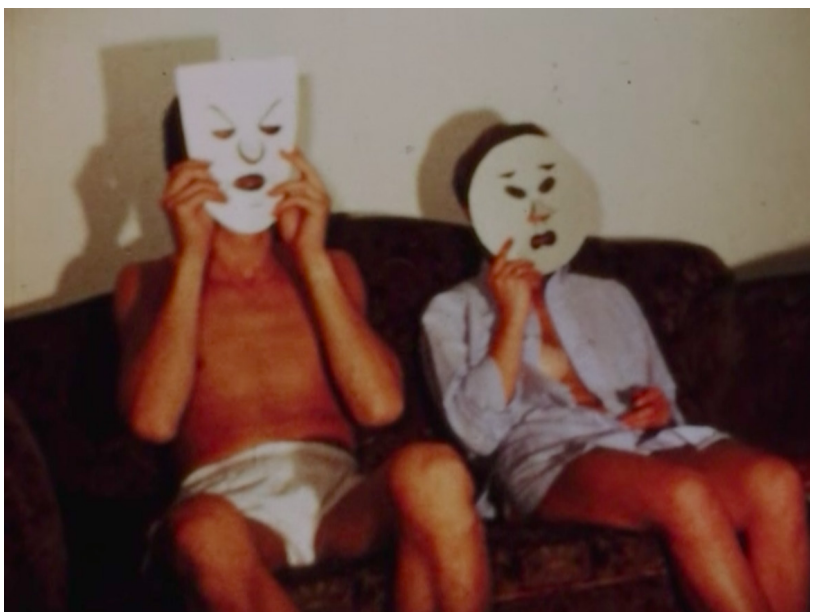

1. Pia Epremian, Proussade, film super8 60', 1967

partire dagli anni Cinquanta. II cinema, dà inoltre la possibilità alle donne di rappresentare sé stesse in modo che i loro stessi corpi, le loro individualità, possano essere guardate e riconosciute senza alcuna idealizzazione né componente desiderante (Melchiori, 1988: 26). II suo linguaggio filmico risente di una certa violenza espressiva e si serve del mezzo cinematografico quasi a scopo terapeutico.

La tesi di laurea in filosofia su Marcel Proust forma la base per il suo primo film intitolato Proussade [1] e realizzato nel 1967 per «liberarsi da Proust», come scrive la stessa Epremian.

Alla base della ideazione di questo lavoro vi è l'indagine sui personaggi della Recherche e sui possibili confronti tra Essere e tempo di Heidegger e la concezione del tem-

2. Pia Epremian, still tratto dal film collettivo Tutto, tutto nello stesso istante, film 16mm 25', 1968

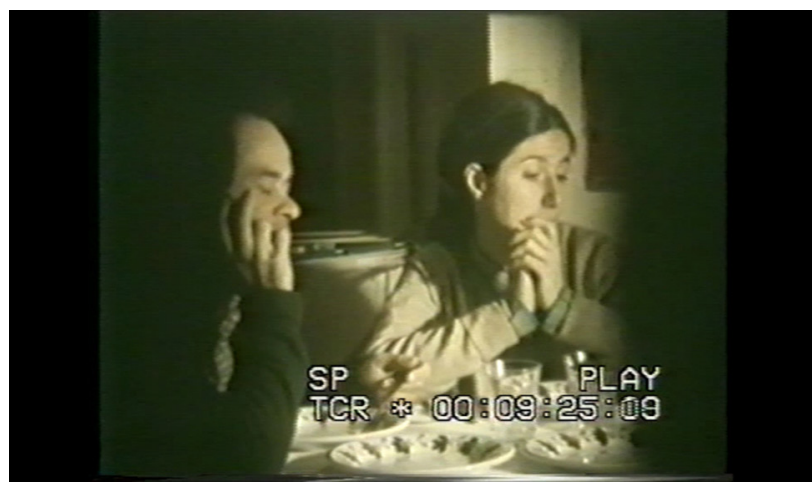

po e dell'essere nell'opera di Proust. II desiderio di portare sullo schermo i personaggi di Proust in un tempo dilatato la spinge a girare questo film. II film, come esplicitato nel titolo Proussade, unisce Proust e Sade per esaltarne le segrete similitudini citando ancora una volta le parole della film-maker.

Nel film ogni persona, come nel mondo personale della Epremian, prende parte alla creazione di una metafora legata alla temporalità proustiana. È evidente già da questa prima opera una inconsueta forza della visione per accedere subito a scelte espressive drastiche. II film, in cui è difficile rintracciare una narrazione, è scandito da rituali compiuti da un uomo e una donna con il volto dipinto di bianco - anche questo un elemento ricorrente in altri film successivi quali Doppio Suicidio (1969) e Antonio delle nevi (1969). Come afferma Lea Vergine, citando la psicanalista Melanie Klein, la dimensione della ritualità, della ripetizione di azioni riparatrici, comporta il prevalere delle pulsioni di vita su quelle di morte (Vergine, 2000: 19). Un altro fattore che caratterizza tutta la sua produzione è l'elemento autobiografico, che diventerà sempre più presente ed evidente nei film successivi.

Del 1968 è il contributo che Epremian invia a Gianfranco Baruchello per il film Tutto, tutto nello stesso istante (1968-1969) [2], un'opera collettiva prodotta dalla già citata Cooperativa Cinema Indipendente di cui Baruchello è l'animatore a Roma ${ }^{8}$.

II documento «collettivo» (Bacigalupo, 1974: 8), così come viene definito da Massimo Bacigalupo, è il risultato da un'operazione priva di qualsiasi finalità estetica: verificare l'esistenza di una qual sintonia in un gruppo abbastanza numeroso (dodici persone) appartenenti alla Cooperativa, come risposta al delicato momento politico e in particolare agli avvenimenti del marzo 1968 presso la facoltà di Architettura a Valle Giulia a Roma (1974: 8). L'arte in quel periodo non volle e non poté tirarsi indietro, si assunse le sue responsabilità politiche dando a queste ultime una forma soprattutto per mezzo della cinepresa, un mezzo diretto e incisivo che si prestava ad una documentazione storica dei fatti politici, oltre che un mezzo artistico. Alla realizzazione del film collettivo partecipano artisti, autori di teatro, del cinema e film-maker quali: Bagigalupo, Baruchello, Bargellini, Chessa, De Bernardi, Epremian, Leonardi, Lombardi, Menzio, Turi, Vergine.

Anche in questo caso la Epremian realizza un film in cui la dimensione performativa e personale è predominante. Lei stessa compare in prima persona, rivolge la telecamera 
3. L'attrice Giuliana Calandra durante la performance La Spia

Ottica di Giosetta Fioroni in occasione del Festival // Teatro delle mostre (Roma, Galleria La Tartaruga, maggio 1968) (foto: Giuseppe Schiavinotto)

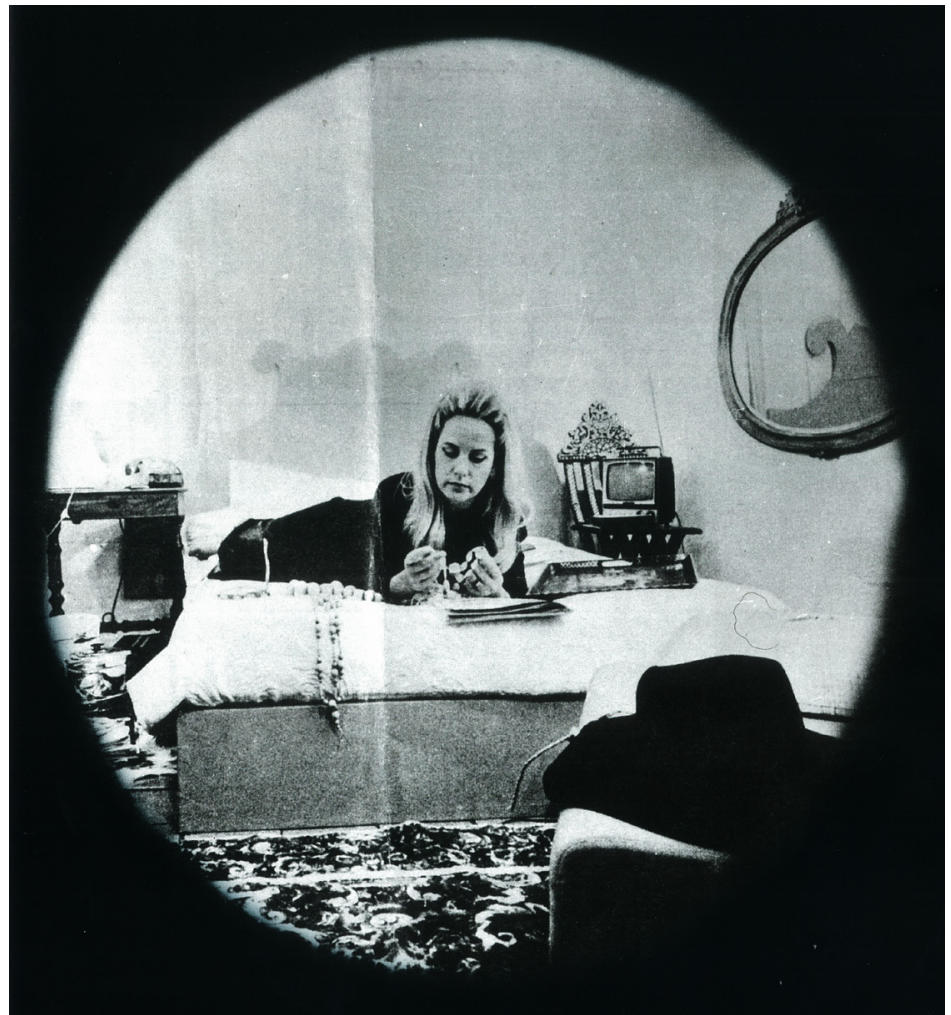

verso di sé, in un'azione oggi molto diffusa con l'utilizzo dei telefoni cellulari. In fase di montaggio l'immagine è sovrapposta alle altre: un accorgimento ricorrente nella cinematografia sperimentale di quegli anni. Lo sguardo della Epremian si avvicina ad una porta e dal buco di una serratura osserva e spia un interno dove si svolgono delle scene ordinarie e intime della vita di una coppia: i due che si svegliano e poi seduti intorno al tavolo da pranzo mentre mangiano. I protagonisti sono Tonino De Bernardi e la moglie Maddalena ripresi in alcuni momenti di intimità familiare.

È da notare come a Roma, nel maggio dello stesso anno, sempre il 1968, Giosetta Fioroni inaugurasse con l'installazione La Spia ottica il festival II teatro delle mostre, presso la Galleria La Tartaruga di Plinio de Martiis, che presentava con cadenza giornaliera e per nove giorni di seguito, un singolo artista con un progetto ambientale o performativo ${ }^{9}$. غ̀ questo uno degli eventi espositivi che segna maggiormente il connubio tra contestazione e sperimentazione, così fortemente presente in quel momento storico. Trasferendo la sua camera da letto in una stanza della galleria, e costringendo i visitatori a sbirciare dal buco di una serratura i gesti caden- zati dell'attrice Giovanna Calandra, Giosetta Fioroni [3], in un processo inverso a quello di Pia Epremian, spettacolarizza la realtà, alludendo ai pionieristici dispositivi del pre-cinema ma anche alle forme allora contemporanee del rotocalco. L'installazione, inserita in una riflessione sulla soggettività femminile, dialoga certamente con la breve esperienza di cinema sperimentale che la Fioroni aveva intrapreso in un periodo circoscritto tra il 1967 e $1968^{10}$. In particolare, nel film la Solitudine femminile del 1967, che precede di poco l'intervento presso la Tartaruga, Giosetta Fioroni sviluppa una narrazione attraverso gesti semplici e ripetitivi, talvolta ossessivi, che denotano il senso di angoscia e inquietudine psicologica della protagonista, impersonata dalla poetessa Rosanna Tofanelli. II ritmo non lineare del film si concentra sulle azioni della protagonista e su una serie di fotografie. il film affonda così nel conflitto tra autenticità e artificio indugiando sul trucco, il travestimento, la maschera, lo specchiarsi in cerca di sé. Una volta truccata e pettinata, la poetessa «viene attraversata da dissolvenze e sovrapposizioni erotico-sensuali» (Celant, 2009: 44) proiettate direttamente sul suo petto, rendendo difficile separare volti proiettati e corpo. 


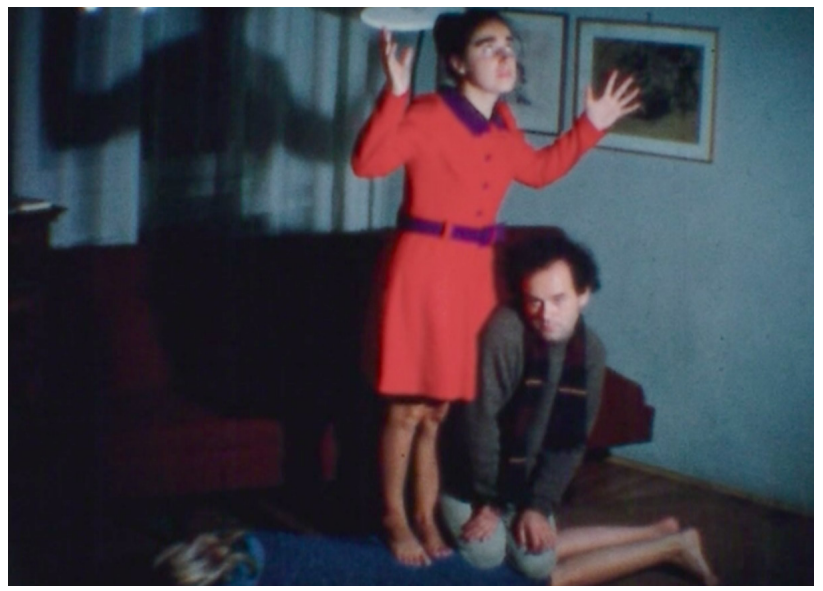

4. Pia Epremian, Medea, film 8mm 25', 1968

In entrambi i casi si possono individuare le tracce di una grammatica teatrale, in cui il gioco del dell'interpretazione fa parte della costituzione in soggetti, come capacità di sdoppiarsi e osservarsi (Passerini, 1988): una dicotomia sulla quale si delineano molte delle opere sperimentali femminili di quegli anni.

II film forse più noto della Epremian, che è stato più volte proiettato anche grazie al soggetto che da sempre ispira scrittori, registi e artisti, è Medea del 1968 [4]. Con questo film il registro espressivo si sposta verso un discorso più aperto, che riconosce il ruolo del singolo individuo nella dimensione più ampia del mito collettivo.

La film-maker realizza questo film dopo la diagnosi della malattia della figlia. Le testimonianze di sé, della propria vita, l'intera sfera del privato vengono impiegate come materiale di repertorio.

\begin{abstract}
Medea raccoglie tutti i personaggi importanti della mia famiglia e dei miei amici. Soprattutto mia figlia Alida bambina quando, ricoverata in ospedale, le scoprono un diabete infantile. II mio sentirmi cattiva madre, trasmettitrice di mali ereditari, mi ha indotto a intitolare il film Medea. Tutti i personaggi in qualche modo simbolicamente trasmettono le pene interiori della nascita, del corpo e della vita ${ }^{11}$.
\end{abstract}

E ancora: «Medea è per me un film sulla morte, ed in primo luogo quella degli altri, che li si ami o no» (Bacigalupo, 1974: 85). Per la regista Medea è l'essere che piange, ama e pro- duce la morte degli altri. Nel film si susseguono scene in cui uomini e donne di età diverse compiono azioni semplici, a tratti banali, come affacciarsi e guardare da una finestra: una ragazza si inginocchia davanti a un albero; una madre chiama a sé una bambina che lo spettatore non vede, ma di cui intuisce la presenza.

Il ruolo di Medea viene interpretato da diversi attori e per sostituzione viene vissuto da più di una persona: la donna giovane, l'anziana. Sebbene siano presenti caratteri maschili, è sempre una donna la protagonista delle scene, in quanto vi è una forte identificazione tra i personaggi e la regista: è il suo film, il suo dramma, la sua vita. È lei che recita e ci mostra sé stessa come Medea; ancora una volta il mondo che viene rappresentato è il suo, con i suoi familiari, il marito, la figlia, gli amici, i conoscenti i suoi luoghi.

In questa pellicola si evidenzia un altro elemento peculiare di questi lavori: come già pubblicava nel 1952 lo psicanalista austriaco Ernst Kris, citato da Lea Vergine: «nel corso degli ultimi decenni le conoscenze psicoanalitiche dei processi di creazione artistica sono esse stesse divenute un fatto artistico [...] la psicoanalisi e le sue scoperte agiscono sull'arte e sull'artista come una forza sociale» (Vergine, 2000: 9). E' forse il caso di segnalare che, una volta conclusa l'esperienza da film-maker, Pia Epremian intraprenderà un percorso professionale come psicoanalista.

Medea viene proiettato a Roma nel 1969 in occasione di una rassegna della Cooperativa Cinema Indipendente presso il cinema Filmstudio ${ }^{12}$, uno dei luoghi promotori del cinema cosiddetto underground italiano, che tra i suoi fondatori annovera Annabella Miscuglio, protagonista dell'attivismo femminista e co-autrice di opere e documentari di inchiesta e di denuncia nella stagione dei collettivi femministi.

Nel film Dissolvimento (1970), gli oggetti della quotidianità casalinga compongono una grammatica della nevrosi. È la storia di due donne che mettono in scena in un happening silenzioso gli eventi della propria esistenza quotidiana. La prima, la pittrice Gigliola Carretti, si pulisce i denti in maniera ripetitiva; la seconda, Epremian stessa, riproduce la condizione umana che necessita di escrezioni e di abluzioni. II film termina con la visione delle cascate delle fontane di Torino. Nelle intenzioni della regista la distinzione tra sublime e volgare non ha senso perché è proprio la distinzione tra dimensione pubblica e privata che viene azzerata. L'azione diviene un modo per riflettere sul corpo e i com- 


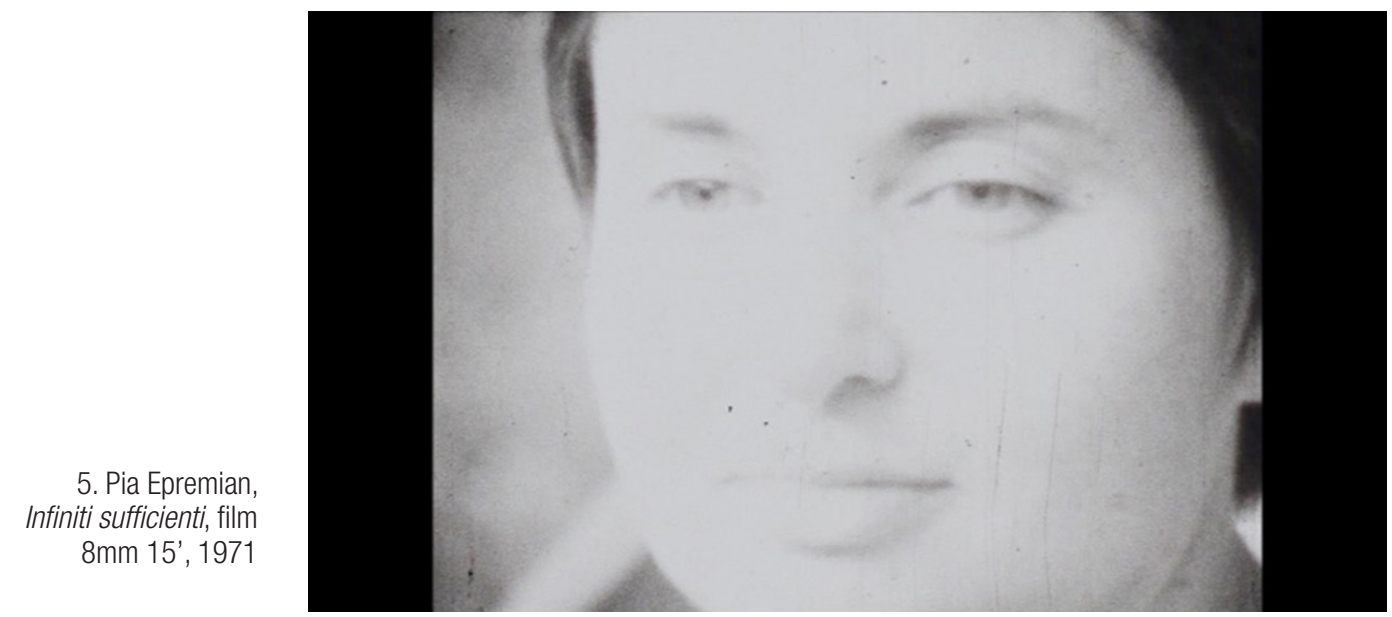

portamenti, sulle norme e i codici culturali che erano stati alla base di concezioni, società e soggettività. II corpo non è più l'ostacolo che separa il pensiero da se stesso, ciò che il pensiero deve superare per arrivare a pensare. Al contrario, è ciò in cui esso affonda o deve affondare per raggiungere l'impensato, cioè la vita (Deleuze, 1989). Come osserva Passerini, il cinema in prima persona esplode come la più felice tra le sperimentazioni di quegli anni, certamente quella che più caratterizza la produzione delle donne in cerca di sé. Le loro opere, poche in una prolifica produzione maschile, segnano la mappa di una nuova identità collettiva in divenire (Passerini, 1988).

In Infiniti Sufficienti (1970) [5], il suo ultimo film, Epremian indaga l'identità racchiusa negli spazi privati della cura familiare e in particolare il rapporto madre e figlia ${ }^{13}$.

Di questo film Straub diceva che era la storia di una donna. Infatti è il racconto di una madre con una bambina che guarda e osserva le altre madri con i loro bambini: all'ufficio sanitario sulle panchine del parco e in ultimo sul balcone una di esse guarda il mondo esterno e me che la riprendo (Bacigalupo, 1974: 87).

Nel film sono ripresi dei ritratti di donne: una donna seduta su una panchina di un giardino pubblico a Torino rappresenta l'alienazione attraverso la propria immobilità e, al tempo stesso, un primo inizio di ricerca di una propria identità. La donna che guarda e ride dal balcone indica nella sua inope- rosità il momento concettuale possibile che la induce a ricercare oltre la corporeità anche il proprio ruolo sociale (1974: 88). In Infiniti Sufficienti è rappresentato il momento di attesa che prelude alla ricerca della realizzazione sociale, politica, culturale ed economica della figura femminile.

In una lettera indirizzata a Gianfranco Baruchello, la Epremian scrive:

Ho fatto questi pochi film (quasi mi vergogno di dire che ho fatto) proprio perché non volevo usare delle parole per dire ciò che poi è venuto fuori, detesto la verbosità filmica e poi il mio era un primo modo di espressione come il vagito di un bambino [...]. Come potrei su questi miei film, potrei dirti femminili i primi, femminista l'ultimo (dove per fortuna c'è già l'abbozzo del pensiero) dire mentre proprio quello che volevo era mostrare! (Bacigalupo,1974: 88).

Queste parole della Epremian sembrano dimostrare come attraverso l'uso di tecniche e linguaggi sovrapposti, in questo "cinema» al confine tra arte e immagine in movimento, la dimensione interiore diveniva presenza e, proprio perché agita dinanzi alla macchina da presa, metteva in gioco, e allo scoperto, dinamiche comportamentali e interiori, altrimenti difficilmente comunicabili. Nel tentativo di spostare la propria in una dimensione quotidiana vissuta e agita in prima persona, sono gli spazi di una nuova soggettività femminile a costituire una delle eredità tra le più significative di quella stagione di sperimentazioni artistiche e culturali. 
Note

1 Viene qui utilizzato il sostantivo film-maker, neologismo entrato in uso nella lingua italiana, per indicare l'autore di un film; differente dal regista in quanto segue in prima persona tutte (o quasi) le fasi di lavorazione dell'opera. Con riferimento a tale estensione della definizione di film-maker come artista faccio riferimento a Mc FADDEN, Cybelle H. (2014), Gendered Frames, Embodied Cameras. Varda, Akerman, Cabrera, Calle, and Maïwenn, Fairleigh Dickinson University Press, Madison.

2 Recentemente queste questioni sono state affrontate con nuove ed interessanti punti di vista in If Arte Povera was Pop Artists' and Experimental Cinema in Italy 1960s-70, programma della rassegna a cura di A. Lissoni, A. Licciardello, S. Toffetti, (Tate Modern Londra, 23-25 Ottobre 2015$)$, Londra 2015.

3 Tra i primi studi che hanno affrontato la storia del film d'artista relativi al panorama italiano si vedano: GRANCHI, Andrea (a cura di) (1978), Cinema d'artista e cinema sperimentale in Italia: 1960-1978, Firenze; FAGONE, Vittorio (1990), L'immagine video, Milano; BORDINI, Silvia (1995), Videoarte e arte. Tracce per una storia, Roma, 1995; DI MARINO, Bruno (1999), Sguardo inconscio azione. Cinema sperimentale e underground a Roma (1965-1975), Roma; MADESANI, Angela (2002), Le icone fluttuanti. Storia del cinema d'artista e della videoarte in Italia, Milano. Fagone organizzò anche alcune rassegne fondamentali sul rapporto tra arte e cinema, si veda tra le altre: Arte e cinema. Per un catalogo del cinema d'artista in Italia, 1965-1976 (Centro internazionale di Brera, 17-21 maggio 1976), Milano, 1976.

4 Sull'argomento che è stato oggetto di studio da parte di numerosi autori italiani si vedano: APRÀ, Aprà (a cura di) (1986), New American Cinema. I/ cinema indipendente americano degli anni Sessanta, Torino; DI MARINO, Bruno (2002), Interferenze dello sguardo. La sperimentazione linguistica tra analogico e digitale, Roma; HIBON, Danièle e BONNEFOY, Françoise (a cura di) (1992), Jonas Mekas, Paris; MEKAS, Jonas (1972), Movie Journal: The Rise of the New American Cinema, 1969-1971, New York.

5 Cito come esempio Molla, il film in 8mm realizzato da Gianfranco Baruchello nel 1960 e recentemente ritrovato presso l'archivio dello ZKM di Karlsruhe. Cit. RABOTTINI, Alessandro e SUBRIZI, Carla (a cura di) (2016-2017), Gianfranco Baruchello. Archive of Moving Images, Milano.

6 Oltre alle interviste pubblicate su quotidiani e riviste online negli anni quali ad esempio PICCINO, Cristina, La ragazza con la macchina da presa in «ll Manifesto", 25 Aprile 2014, <https://ilmanifesto.it/pia-epremiam-la-ragazza-col-super8/>, si fa riferimento alla trascrizione di una conversazione avvenuta nello studio di Pia Epremian nel febbraio 2018.

7 I primi incontri per la costituzione della Cooperativa Cinema indipendente avvengono a Roma nel maggio del 1967 tra i rappresentanti dei gruppi di Torino e di Roma. La costituzione formale e la relativa registrazione avviene a Napoli nello stesso anno per iniziativa di Adamo, Aldo e Antonio Vergine. II catalogo della Cooperativa Cinema Indipendente è stato consultato presso l'archivio della Fondazione Baruchello. Sul documento del 1967 sono elencati i seguenti film-makers e artisti: Angeli, Bacigalupo, Baruchello, Bignardi, Capanna, De Bernardi, De Rinaldo, Dogliani, Elia, Epremian, Ferrero, Grifi, Leonardi, Loffredo, Mantelli, Menzio, Oriani, Patella, Serna, Siniscalchi, Turi, i fratelli Vergine.

8 Dopo il primo periodo di attività a Napoli, nel 1968 la sede della Cooperativa Cinema indipendente si trasferisce a Roma in Via Monte Senario 23.

9 || «Festival» come viene definito nel catalogo originale edito da Lerici editore, si tenne a Roma da lunedi 6 al 31 maggio 1968 e vide la partecipazione degli artisti: Franco Angeli, Nanni Balestrini, Alighiero Boetti, Sylvano Bussotti, Pier Paolo Calzolari, Enrico Castellani, Mario Ceroli, Ciro Ciriacono, Giosetta Fioroni, Laura Grisi, Ettore Innocente, Renato Mambor, Gino Marotta, Fabio Mauri, Giulio Paolini, Emilio Prini e Paolo Icaro, Paolo Scheggi, Loreto Soro, Goffredo Parise, Cesare Tacchi.

10 I film di Giosetta Fioroni al momento reperibili presso l'archivio dell'artista sono: La Solitudine femminile (1967, 8mm, b/n, 8'); Gioco (1967, 16mm, b/n, 6'); Coppie (1967, 16mm, b/n, 15'); Goffredo (1967, 8mm, b/n, 6'). Sui film di Giosetta Fioroni si vedano anche: C. Casero, Giosetta Fioroni. Oltre il dipingere, in «Arabeschi», n.8 luglio-dicembre 2016 http://www.arabeschi.it/giosetta-fioroni-oltre-il-dipingere/; SIMl, Giulia (2015), «Rivoluzioni in cerca di sé: il Sessantotto intermediale di Giosetta Fioroni», in CARDONE, Lucia e FILIPPELLI, Sara (a cura di), Filmare il femminismo. Studi sulle donne nel cinema e nei media, Pisa, ETS, pp. 233-243; VOLPATO, Elena (1999), «l film di Giosetta Fioroni», in SPADONI, Claudio (a cura di), Giosetta Fioroni, catalogo della mostra (Ravenna Pinacoteca Comunale Loggetta Lombardesca, 1999-2000), Mazzotta, Milano.

11 Si fa riferimento alla trascrizione della conversazione con la film-maker avvenuta presso il suo studio nel febbraio 2018, anche in questo caso le informazioni sono riportate nel capitolo a lei dedicato nel-n.s sul cinema sperimentale italiano -in «Bianco e nero », cit.-, p. 85

12 La sala cinematografica Filmstudio viene fondata a Roma nell'ottobre del 1967 da Amerigo Sbardella, per notizie e cronache della programmazione si rimanda nuovamente a Bacigalupo (1974).

13 Testo inedito di S. Traversa, consultato presso l'archivio di Pia Epremian.

\section{Bibliografia}

BACIGALUPO, Massimo (1974), «\|l Film Sperimentale», Bianco e Nero, vol. XXXV, n. ${ }^{\circ}$ 5/8, maggio-agosto.

CELANT, Germano (1976), Precronistoria. 1966-69. Minimal Art, pittura sistemica, arte povera, land art, conceptual art, body art, arte ambientale e nuovi media, Centro Di, Firenze.

- (2009), Giosetta Fioroni, Skira, Milano.

DE BERNARDI, Tonino (2017), «Cinéma expérimental en Italie: fin de annés 60», in LICCIARDELLO, Annamaria, Cinéma expérimental underground italien. Les annés soixante, La Coopèrative de Rome, Marseille.

DELEUZE, Gilles (1985/1989), L'image-temps, Cinéma 2, Les Éditions de Minuit, Paris, prima edizione italiana: 1989, Ubulibri, Roma.

DI MARINO, Bruno (1999), Sguardo inconscio azione: cinema sperimentale e underground a Roma 1965-1975, Lithos, Roma. 
FAGONE, Vittorio (2008), «Jonas Mekas e il New American Cinema», in NORTHOVER, Benn (a cura di), 6 Opere di Jonas Mekas, Fondazione Raggianti, Lucca, pp. 13-17.

JONES, Amelia (1998), Body Art. Performing the subject, University of Minnesota Press, Minneapolis.

- (2006), Self/Image: technology, representation and the contemporary subject, Routledge, London.

MC FADDEN, Cybelle H. (2014), Gendered Frames, Embodied Cameras. Varda, Akerman, Cabrera, Calle, and Maïwenn, Fairleigh Dickinson University Press, Madison.

MELCHIORI, Paola (1988), «Women's Cinema: A Look at Female Identity», in BRUNO, Giuliana e NADOTTI, Maria (a cura di), Off Screen: Women and Film in Italy, Routledge, London/New York, pp. 25-35.

MULVEY, Laura (1989/2009), Visual and other pleasures, Palgrave Mcmilan, Basingstoke.

PASSERINI, Luisa (1988), Autoritratto di gruppo, Giunti Barbèra, Firenze.

PERNIOLA, Mario (2009), Miracoli e traumi della comunicazione, Einaudi, Torino.

VERGINE, Lea (1974/2000), /l corpo come linguaggio, Skira, Milano.

WILLENER, Alfred (1970), L'image-action de la societé ou la politisation culturelle, Éditions du Seuil, Paris. 